\title{
Chronic Recurrent Multifocal Osteomyelitis in Children: A Single Center Experience
}

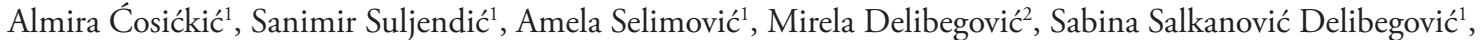
Evlijana Zulićn ${ }^{1}$ Dina Delibegović ${ }^{1}$, Damir Sabitović ${ }^{3}$, Fejzo Džafić ${ }^{3}$

${ }^{1}$ Clinic for Children's Diseases Tuzla, University Clinical Centre Tuzla, Bosnia and Hezegovina, ${ }^{2}$ Clinic for Radiology and Nuclear Medicine, University Clinical Centre Tuzla, Bosnia and Hezegovina, ${ }^{3}$ Polyclinic for Laboratory Diagnostics University Clinical Centre Tuzla, Bosnia and Herzegovina
\end{abstract}

Correspondence: almiracosickic@gmail.com; Tel.: + 387611944 04; Fax.: + 38735303740

Received: May 21, 2020; Accepted: August 9, 2020

\begin{abstract}
Objective - To evaluate the suitability of diagnosing chronic recurrent multifocal osteomyelitis (CRMO) according to the Bristol diagnostic criteria and the clinical outcome of the children included in the study. Materials and Methods - Retrospectiveprospective study was conducted at the Clinic for Children's Diseases, University Clinical Center Tuzla in the period from January 2018 to January 2020. The medical records of children treated CRMO were analyzed. Results - Eight children fulfilled the Bristol diagnostic criteria. The median age at disease onset was 10.7 years. All children had multifocal lesions which relapsed in 2 children, and predominantly affected regions were the pelvis, hips, femur, spine and shoulder girdle. Hematological and biochemical parameters were unremarkable, although ESR was elevated in $6 / 8$ children; all children had $\mathrm{CRP}<30 \mathrm{mg} / \mathrm{L}$. For 3 children lesions on plain radiography were observed; 49 lesions were verified on MRI ( 4 children had whole body MRI). Bone biopsy was performed in 2 children and it showed inflammatory changes. In 6/8 children treatment with NSAIDs was sufficient to control the disease during the 8-month period. However, two children had pain resistant to NSAID therapy, so they were treated with methotrexate and sulfasalazine. The child who received sulfasalazine treatment relapsed, so TNF $\alpha$ inhibitor (adalimumab) was used to control disease activity. No child received pamidronate. Conclusions - Our results showed that the use of the Bristol diagnostic criteria may obviate the need for a biopsy, shorten the time of diagnosis, save the bone from destruction, and avoid unnecessary treatments.
\end{abstract}

Key Words: Chronic • Osteomyelitis • Children.

\section{Introduction}

Chronic recurrent multifocal osteomyelitis (CRMO) is a bone disease of unknown origin that mostly affects children and adolescents (1). It seems that CRMO best fits into the category of autoinflammatory disorders, a group of innate immune system disorders in which there are "seemingly unprovoked" episodes of inflammation (2-4). Clinical presentation includes bone pain (typically worse at night) with or without fever. Tenderness, swelling and warmth are often present in the affected bone (5-7). The disease may affect virtually any bone of the body, but the metaphyseal regions of the long bones, clavicle, vertebral bodies and pelvis are the most commonly affected sites (6, 9-13). Additional inflammatory conditions predominantly affecting the skin (acne, palmoplantar pustulosis, psoriasis) and the gut (Crohn disease, ulcerative colitis) are frequent as well $(4,11)$. Non-infectious arthritis can be present in up to $30 \%$ of children, and in up to $60 \%$ of children (mostly adolescents) may resemble or evolve into spondyloarthropathies $(12,15,16)$. Routine inflammatory parameters: white blood cell count (WBC), C-reactive protein (CRP), erythrocyte sedimentation rate (ESR) are 
usually normal or mildly elevated since high titers autoantibodies are typically absent and there is no strong association with HLA-B27 (14, 17). Diagnostic path of CRMO is complicated due to the overlap of clinical and imaging findings. It often remains a diagnosis of exclusions between tumors (Ewing's sarcoma, osteosarcoma, Langerhans cell histiocytosis-LCH) and infectious arthritis (17, 18). Unfortunately, there are various diagnostic criteria for CRMO according to different authors; the latest criteria were made by Roderick et al. (19) (the so-called the Bristol diagnostic criteria for CRMO).

The aim of study was to evaluate the suitability of diagnosing CRMO according to the Bristol diagnostic criteria and the clinical outcome of the children included in the study.

\section{Patients and Methods}

This retrospective-prospective study was conducted at the Department of Rheumatology, Immunology and Allergy of the Clinic for Children's Diseases, University Clinical Center (UCC) Tuzla in the period from January 2018 to January 2020. The medical records of children with CRMO were analyzed. The diagnosis of CRMO was made based on the Bristol diagnostic criteria: the presence of typical clinical findings (bone pain with or without localized swelling, without significant local or systemic features of inflammation or infection) and the presence of typical radiological findings (plain radiography showing combination of lytic areas, sclerosis and new bone formation, or preferably magnetic resonance imaging (MRI) showing bone marrow oedema with or without bone expansion, lytic areas and periosteal reaction). This finding associated with: criterion 1) more than one bone (or clavicle alone) without significantly raised $\mathrm{CRP}<30$ $\mathrm{g} / \mathrm{L}$ or criterion 2) if unifocal disease (other than clavicle), or CRP>30 g/L, with bone biopsy showing inflammatory changes (plasma cells, osteoclasts, fibrosis or sclerosis) with no bacterial growth whilst not on antibiotic therapy were taken into account to establish the diagnosis as well (19). The exclusion criteria were the unfulfilled Bristol diagnostic criteria for CRMO, infectious or hematologic diseases. The following were analyzed: gender, age of children when first symptoms occurred, age at diagnosis, interval from onset of symptoms to diagnosis, number of painful and sensitive regions (pain intensity rated $0-10$ by the visual analogue scale-VAS for child/parent assessment), manifestations such as impaired mobility, general clinical signs (malaise, fatigue, fever, loss of appetite, weight loss), skin changes, symptoms of other organ systems, associated disease/condition, family history, effects of previously conducted treatments. Hematological and biochemical test results were considered as normal: ESR $\leq 15 \mathrm{~mm} / \mathrm{h}, \mathrm{CRP}<5$ $\mathrm{mg} / \mathrm{L}$, WBC 4.4-11.60x10\%/L, fibrinogen 1.8-3.5 $\mathrm{g} / \mathrm{L}$, antinuclear antibody-ANA (Elisa Hytec) with cut off $23 \mathrm{IU} / \mathrm{ml}$.

Serology tests were analyzed (enzyme linked immunosorbent assay-Elisa) detecting the presence of antibodies (cut off $25 \mathrm{IU} / \mathrm{ml}$ ) against Epstein Barr virus (EBV), Borellia burgdorferi, Brucella species, Chlamydia and Mycoplasma pneumoniae, antibody titers (nephelometry) to antistreptolysin O (ASO) and anti Deoxyribonuclease B (anti DNaseB) with cut off $200 \mathrm{IU} / \mathrm{ml}$. We assessed microbiological analysis results (blood culture, the culture of biopted tissue), radiological examination results (plain radiography and magnetic resonance imagingMRIsymptomatic region, MRI Whole Body-WB MRI), histological findings of the biopted materials, and the effects of the therapy applied. During the two-year follow-up the quarterly examinations were conducted, which included assessment of a number of painful regions, pain intensity by VAS, general clinical signs, skin changes, symptoms of other organ systems and CRP, ESR, MRI of the affected regions.

\section{Ethics Statement}

The study protocol was approved by Ethics Committee of UCC Tuzla, No 01/1-37-492/18. Informed consent was signed by parents of all participants. 


\section{Statistical Analysis}

Statistical data analysis was conducted using the biomedical software application "MedCalc for Windows, Version 15.11.4"(MedCalc Software, Ostend, Belgium). The variables with distorted distribution were shown with median as a measure of the central value. The Mann- Whitney test was used to test the statistical significance of the difference between the samples, including plain radiography findings and duration of symptoms. Pearson's correlation coefficient was used to assess correlation of variables such as MRI findings and duration of symptoms. The difference was considered significant when $\mathrm{P}<0.05$.

\section{Results}

Four out of 12 children with suspected CRMO met the exclusion criteria, i.e. two with infectious osteomyelitis, one had leukemia and one had osteoid osteoma. Thus, eight children were included in the study, six boys and two girls, whose median age at disease onset was 10.7 years (minimum and maximum from 7.6 to 12.1 years). The mean median diagnostic delay was 9 months (minimum and maximum from 3.5 to 25.5 months). The children experienced pain and limited mobility in their lower extremities (seven children), their upper extremities and shoulder girdle (two children), their lower back and hips (seven children), and/or in their back (five children); one child had clavicular pain and swelling. Six out of eight children had a history of multifocal or changing complaints, and the VAS pain intensity, which ranged from 6-9. Initial hematological and biochemical parameters were unremarkable, although ESR was elevated in six out of eight children (median $26 \mathrm{~mm} / \mathrm{h}$, minimum and maximum from 22 to $58 \mathrm{~mm} / \mathrm{h}$ ), all children had $\mathrm{CRP}<30 \mathrm{mg} / \mathrm{L}$ (median value of $6.4 \mathrm{mg} / \mathrm{L}$, minimum and maximum from 5.2 to $10.3 \mathrm{mg} / \mathrm{L}$ ). The values did not increase significantly during the treatment and follow-up period. Blood culture was negative in all children. None of the children clinicall or serologically showed signs of acute infection (EBV, Borrelia burgdorferi,
Brucella, Chlamydia and Mycoplasma pneumoniae); four children had elevated ASO and Anti-DNase B titers. One child had compromised neutrophil function-decreased chemotaxis and phagocytic activity of neutrophils. General symptoms were present in $6 / 8$, skin lesions (pyoderma, acne) in $2 / 8$, while pain and swelling (without heat) of the joints developed in 5/8 children. Family history of autoimmune diseases was positive in two children: a father of one child (with acne conglobata) had acne vulgaris and a mother of another child had juvenile idiopathic arthritis (JIA) in childhood. 5/8 of children other comorbidities. The clinical characteristics of children are shown in Table 1.

Plain radiograph of the major symptomatic site was abnormal in $3 / 8$ of children (a combination of lytic areas, areas of bon sclerosis, and periosteal reaction) and there were 16 silent lesions as well. The median duration of symptoms was 7.6 months in five with normal radiograph findings, but 8.6 months in children with pathologic radiograph findings. There were no statistically significant differences $(\mathrm{P}=0.39)$ in the duration of symptoms between the children with normal and pathologic radiographs. MRI identified 49 lesions (bone marrow edema, bone expansion, lytic areas and periosteal reaction) in eight children, four of whom had whole body MRI. The number of lesions verified by MRI is shown in Table 2. Seventeen out of 49 $(34.7 \%)$ lesions were silent and identified in five children who had normal plain radiographs. All children had multifocal lesions, two of them had relapsing lesions; three children had multiple lesions of one particular bone (femur, sacrum, iliac bones).

There was no statistically significant association between the duration of symptoms and a number of verified lesions by MRI ( $r=0.15, \mathrm{P}=0.714 ; 95 \%$ CI: -0.61-0.77). Four out of eight children who had MRI lesions on iliac bones and/or sacrum had bilateral effusions in the sacroiliac (SI) joints; one girl had talocrural (TLC) joint effusion.

Bone biopsy (clavicle, tibia) was performed in two children, who had been treated by an orthopedist before they were admitted to our department. 


\begin{tabular}{|c|c|c|}
\hline \multicolumn{3}{|c|}{$\begin{array}{l}\text { Table 1. Clinical Characteristics of Eight Children } \\
\text { Included in the Study }\end{array}$} \\
\hline Children's characteristics & & $\mathrm{N}$ \\
\hline \multirow{2}{*}{ Gender } & Male & 6 \\
\hline & Female & 2 \\
\hline \multirow{2}{*}{ Swelling above the affected bones } & No & 6 \\
\hline & Yes & 2 \\
\hline \multirow{2}{*}{ Arthritis } & No & 3 \\
\hline & Yes & 5 \\
\hline \multirow{8}{*}{ Changes in general condition } & TLC $^{*}$ joint & 1 \\
\hline & $\mathrm{SI}^{\dagger}$ joint & 4 \\
\hline & No & 2 \\
\hline & Yes & 6 \\
\hline & Weakness & 6 \\
\hline & Loss of appetite & 4 \\
\hline & Loss of body weight & 2 \\
\hline & Febrility & 4 \\
\hline \multirow{4}{*}{ Skin changes } & No & 6 \\
\hline & Yes & 2 \\
\hline & $\mathrm{PG}^{\ddagger}$ and $\mathrm{AC}^{\varsigma}$ & 1 \\
\hline & Granuloma faciei & 1 \\
\hline \multirow{2}{*}{$\begin{array}{l}\text { Family history of autoimmune } \\
\text { diseases }\end{array}$} & No & 6 \\
\hline & Yes & 2 \\
\hline \multirow{9}{*}{ Associated diseases/conditions } & No & 3 \\
\hline & Yes & 5 \\
\hline & JIA" & 1 \\
\hline & $\mathrm{IBD}^{9}$ & 1 \\
\hline & BHS $^{* *}$ & 4 \\
\hline & $\mathrm{CHD}^{\dagger \dagger}$ & 1 \\
\hline & Epilepsy & 1 \\
\hline & Enuresis nocturna & 1 \\
\hline & Asthma & 1 \\
\hline
\end{tabular}

"Talocrural joint; Sacroiliac joint; ${ }^{\ddagger}$ Pyoderma gangrenosum; ${ }^{\S}$ Acnae conglobata; "Juvenile idiopathic arthritis; 'Inflammatory bowel disease; "Benign hypermobile syndrom; ${ }^{\dagger}$ Congenital heart defects.

Infiltration fields with mononuclear cells, plasma cells and lymphocytes with signs of focal edema of fibrous stroma were observed in the analyzed samples. In the bioptate of clavicle tissue, granulomas composed of polymorphonuclear leukocyte clusters with macrophages were noticed. The cultures of biopted tissue were negative for the presence of bacteria or fungi.
Table 2. Distribution of Lesions ( $\mathrm{N}=49$ ) Verified by MRI

\begin{tabular}{ll}
\hline Localization of lesions & $\mathrm{N}(\%)$ \\
\hline Iliac bones & $13(26.53)$ \\
\hline Femur & $11(22.44)$ \\
\hline Tibia & $3(6.12)$ \\
\hline TLC joint $^{\dagger}$ & $2(4.08)$ \\
\hline Radius $^{\dagger}$ & $4(8.17)$ \\
\hline Mandible & $2(4.08)$ \\
\hline Clavicula & $1(2.04)$ \\
\hline Ribs & $4(8.17)$ \\
\hline Vertebrae & $9(18.37)$ \\
\hline Total & $49(100)$ \\
\hline
\end{tabular}

"Magnetic resonance imaging; ${ }^{\dagger}$ Talocrural joint.

After infectious and hematologic diseases had been excluded, eight children fulfilled the Bristol diagnostic criteria; all children fulfilled the first diagnostic criterion, the presence of typical clinical findings: eight children had bone pain, while two of them had localized swelling. None of the children had significant local or systemic features of inflammation or infections. The second criterion was fulfilled as well: three children had the presence of typical radiological findings on plain radiograph, a combination of lytic areas, sclerosis and new bone formation. However, all children had bone marrow edema with or without bone expansion, lytic areas and/or periostal reaction on MRI, multifocal lesions and the values of CRP $<30 \mathrm{mg} / \mathrm{L}$.

All children had received at least one course of intravenous antibiotics before the diagnosis was made. Three children were treated with corticosteroids, but none of them responded to the therapy. The treatment with nonsteroidal anti-inflammatory drugs (NSAIDs) was started in all children: naproxen $15 \mathrm{mg} / \mathrm{kg} /$ day or indomethacin $1 \mathrm{mg} / \mathrm{kg} /$ day.

During the two-year follow-up in $75 \%$ of children, treatment with NSAIDs was sufficient to control the disease during the eight month period; those children did not require therapy other than NSAIDs since they were symptom free and with no limitations in movement. However, for three of them MRI showed the progression of old, but also five new lesions. Therefore, for those children 


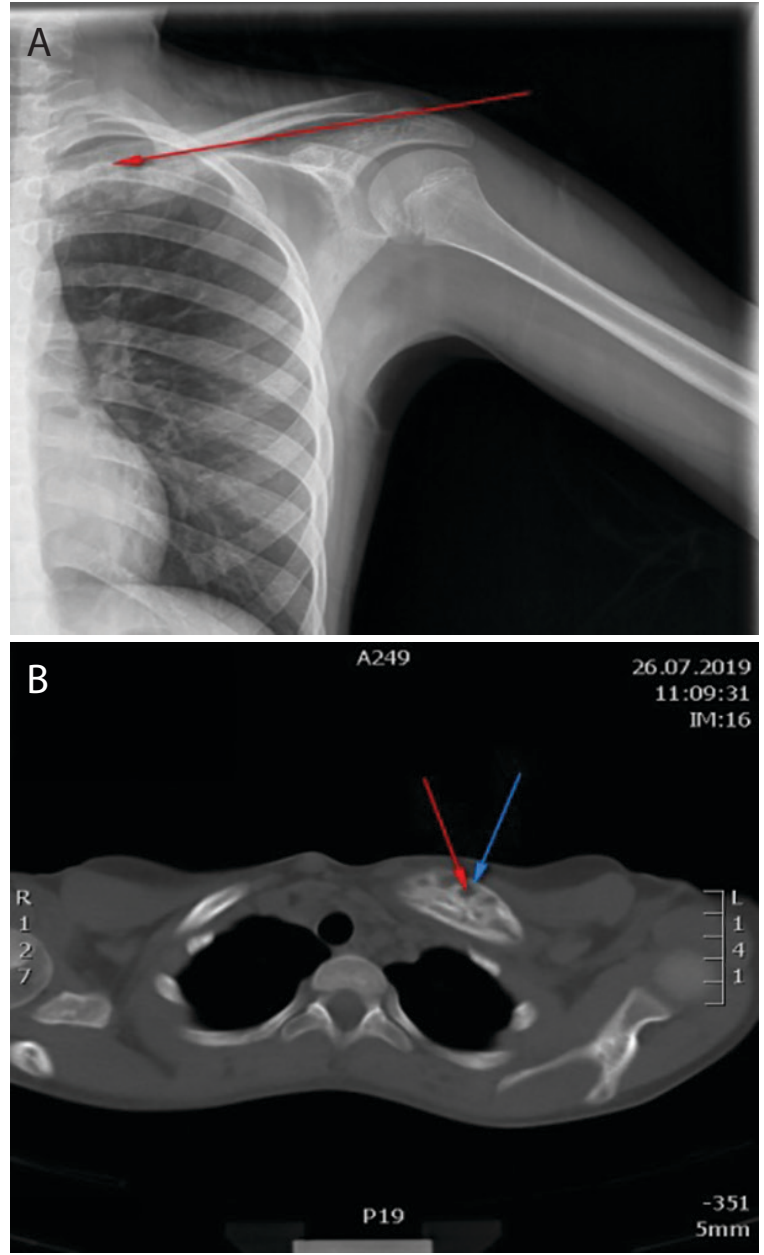

Fig. 1A-B. Plain radiograph and computed tomography of left clavicle-12 year old girl. A: Plain radiograph showed an expanded medial aspect of the clavicle, inhomogeneous structure with lytic lesions and irregular oval sclerosis (red arrow); B: Computed tomography scan showed medial aspect of the clavicle expanded with visible multiple lytic lesions (red arrow) and surrounding sclerosis (blue arrow).

the treatment with naproxen was continued. Three months later, new MRI findings showed regression of lesions in two children, while one child was lesion free, while one child was lesion free, therefore after a mean period of 11.3 months the treatment with naproxen was discontinued. Two boys were NSAIDs resistant. One of them was treated with methotrexate (MTX), while the other one was treated with sulfasalazine since he developed inflammatory bowel disease (IBD) during the follow
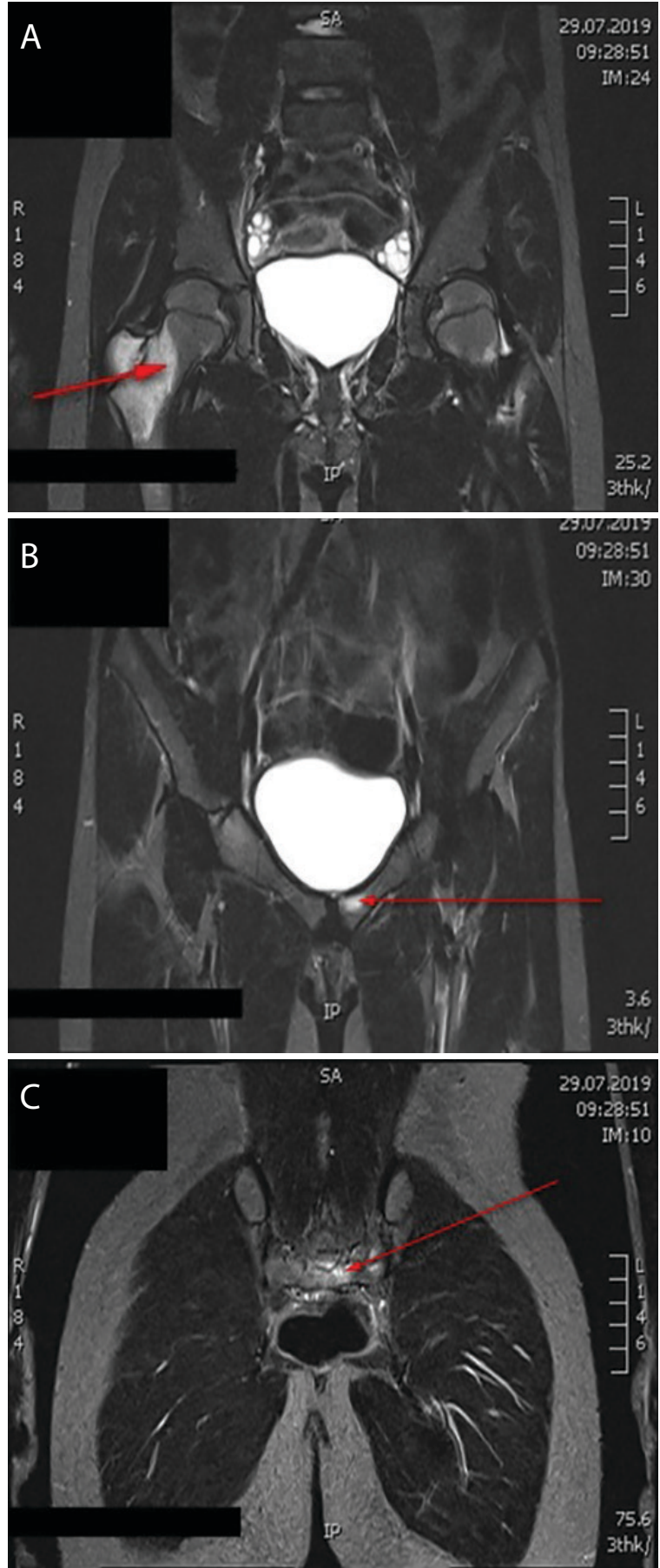

Fig. 2A-C. Magnetic resonance imaging T2STIR pelvic bones-12 year old girl. A: Extensive zones of bone edema of the femoral metaphysis and the great trochanter apophysis (red arrow); B: Focal edema of the upper ramus of the left pubic bone (red arrow); $C$ : bone edema left in the mass of the lateral sacrum (red arrow). 


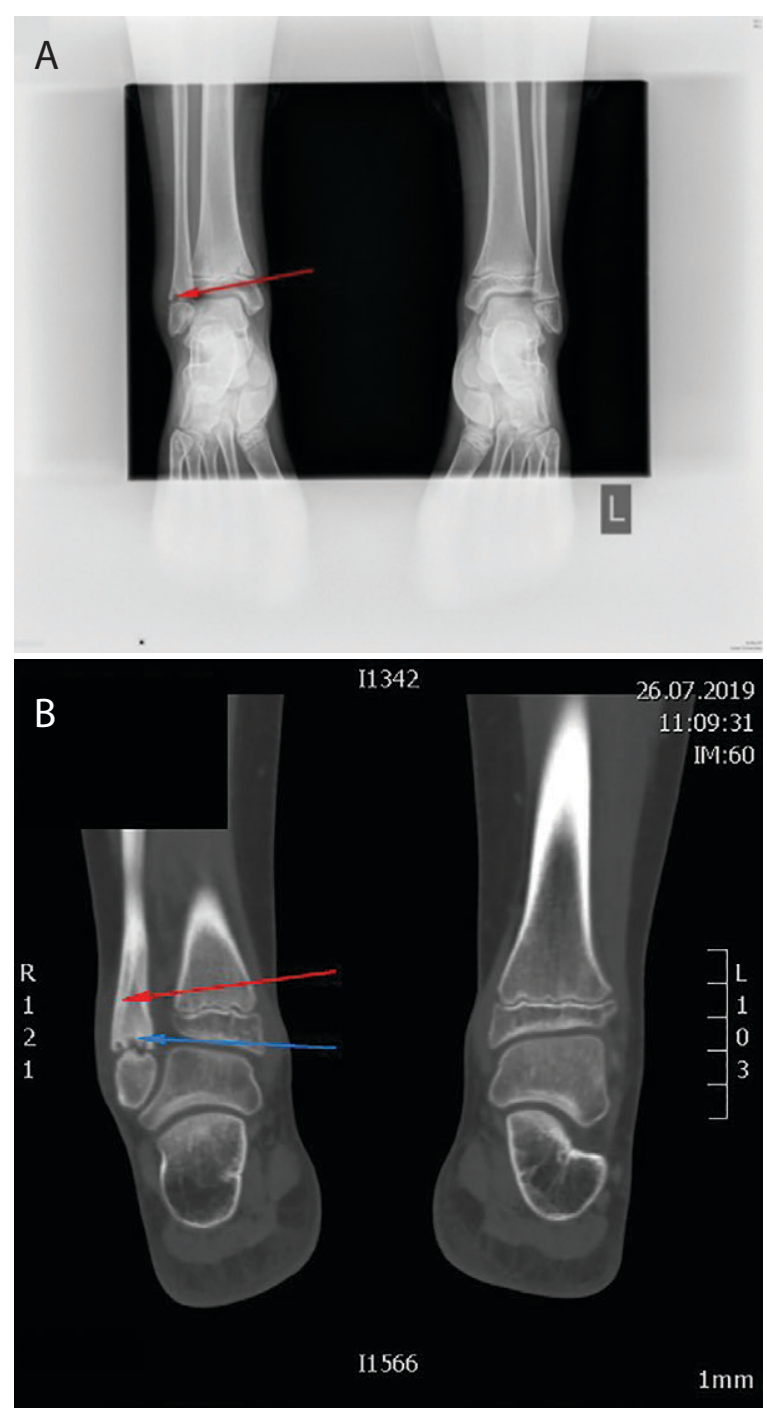

up period. The boy treated with MTX went into remission, while the boy treated with sulfasalazine relapsed at the time of followup. Therefore, TNF $\alpha$ inhibitor (adalimumab) $40 \mathrm{mg}$ sc every 15 days was used in attempt to control disease activity. At the time of writing the article, he was still receiving the aforementioned therapy. No child received pamidronate.

\section{Discussion}

Chronic recurrent multifocal osteomyelitis (CRMO) is primarily a disease of young girls (female to male ratio of 4:1) with the peak onset

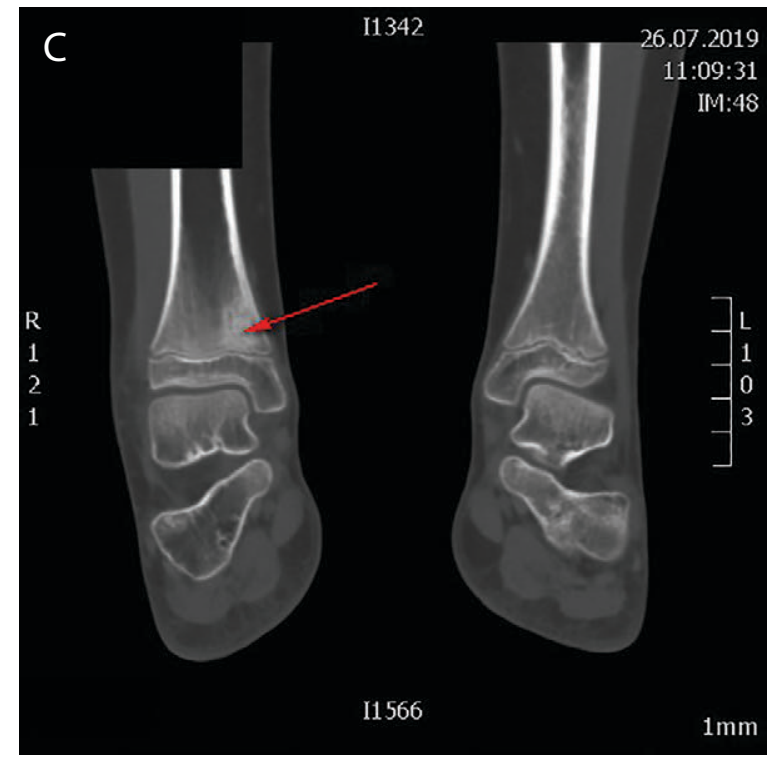

Fig. 3A-C. Plain radiography and computed tomography of the ankles of a 12 year old girl. A: Plain radiography showed in the metaphysis of the right fibula irregular transparency with an interruption in the continuity of the bone towards the epiphyseal fissure, may correspond to lytic lesions with uzura (red arrow); B-C: Computed tomography scan showed periosteal reaction on the metaphysis of the fibula (red arrow) with extension to the epiphysis of the lytic lesion with uzura (blue arrow) (B); On the metaphysis of the right tibia lytic lesion with reactive sclerosis and mild thickening of the cortex (red arrow) (C).

between 7 to 12 years of age $(9,14)$. However, in our study there were more boys than girls and the median age of onset was 10.7 years. In spite of being described in 1972 by Gideon (20), CRMO is still not well recognized and is likely to be substantially more common than is currently diagnosed. The time delay in making the diagnosis is various, so von Kalle et al. (21) reported that the time interval between the first, sometimes insidious, onset of clinical signs and the diagnosis of CRMO ranged from two days to seven years. However, Kaiser et al. (22) found that the mean diagnostic delay in the group with unifocal disease was 12.1 months, while in the group with multifocal onset was seven months. The researchers explained that possible reasons included the fact that plain radiographs were not sensitive enough to detect osteomyelitis or unifocal lesions were misdiagnosed as acute infec- 
tious osteomyelitis. Girschick et al. (11) reported a significant diagnostic delay of approximately one year. Our results were somewhat different- the median diagnostic delay was nine months. The consistent feature of CRMO is the insidious onset of pain with swelling and tenderness localized over the affected bones (19, 23-25). A significant number of patients had a history of multifocal or changing complaints. The metaphyses and epiphyses of the femur, tibia or humerus are the most frequently affected, while involvement of clavicle, sternum or jaw is highly suggestive of a CRMO diagnosis (11, 26). In research by Falip et al. (27) and Gicchino et al. (28) the most common sites were the long bone metaphyses especially femoral and tibial, while slightly fewer lesions were found on pelvis, spine and only $25 \%$ had lesions on clavicle. The pelvis is a typical site of CRMO with $11-34 \%$ of patients affected $(9,29,30)$. That is consistent with the findings of Kaiser et al. (22) who found pelvic lesions in $25 \%$ patients, as well as with our findings, since we found that the most common localization of lesions (44\% lesions) was pelvis; metaphyses and epiphyses of the femur were the second common localizations (22.5\% lesions) in our study; one girl had a lesion on clavicle. The results were somewhat different from Surendra and Shetty (31). In their study, children had more lesions on spine, feet and ribs and the least on the femur, $12.5 \%$ lesions. The researchers showed that lesion may occur in any bone, including vertebrae, and sacroiliitis may also occur as part of the osseous pelvic lesions; arthritis of adjacent and distal joints could manifest in up to $30 \%$ of patients $(6,11,31,32)$. However, in the study by Gicchino et al. (28) $80 \%$ of their patients had arthritis, while somewhat different results were reported by Kaiser et al. (22) who found arthritis in $15 \%$ of patients, five of whom had sacroiliitis. Our results were in accordance with those, $18.4 \%$ of children had lesions of lumbar and sacral vertebrae; four children with lesions on sacrum and iliac bones developed sacroiliitis., and only one girl had arthritis of the TLC joint.

Routine inflammatory parameters (WBC, CRP, ESR) are usually normal or mildly elevated, and the most common abnormal laboratory test is an elevated ESR $(11,12,33)$. All children in our study also had moderate elevated ESR, while the CRP and WBC levels were unremarkable. On the other side, Ferguson et al. (34), Catalano-Pons et al. (35) reported that inflammatory markers increased in more than a half of the examined patients.

The precise immunologic basis of CRMO remains unknown. There is no evidence of immune deficiency in the vast majority of children and the lack of high titer autoantibodies suggests that it does not have an autoimmune basis $(14,10)$. Although a German cohort was noted to have a positive ANA in approximately one third of cases, this was not found in other cohorts (14). Even though there seems to be a clinical overlap, the presence of HLAB27 does not seem to be a hallmark of CRMO (11). In our study, two children were ANA and HLA B27 positive, one of them developed JIA (the group of spondyloarthropathies). There are reports of neutrophil dysfunction in CRMO, however, the role of neutrophils in these disorders has not been fully studied $(32,36)$. In our study, one child had neutrophil dysfunction, decreased chemotaxis and phagocytic activity of neutrophils.

Imaging techniques are centrally important for diagnosing CRMO and excluding differential diagnoses. Inflammatory bone lesions may be detected in plain radiographs as radiolucent, osteolytic, or sclerotic lesions, but may remain normal in early stages (34, 37-39). Particularly in early disease, MRI techniques are highly sensitive, they can detect bone edema even before bone erosions and sclerosis develop, and can help assess inflammation of surrounding tissues. In the study by Kaiser et al. (22) $4 / 41$ patients initially had a normal radiograph of the symptomatic region, but the MRI showed typical findings of marrow edema with hypointense lesions in these patients. Similarly, in our study, MRI showed edema and periostal reaction in five children with normal radiograph on symptomatic region. Falip et al. (27) reported on the radiologic appearance normal, mixed lytic, and sclerotic, sclerotic or lytic lesions in $30.3 \%, 35.7 \%, 14.3 \%$ and $10.7 \%$ of patients, respectively. Hofmann et al. (40) found 
that the most common MRI finding was medullary edema (42 patients), epiphyseal or soft-tissue inflammation in $52.4 \%$ and $16.6 \%$ of patients, respectively. Our results were somewhat similar, $37.5 \%$ of children had characteristic radiographic lesion of the symptomatic region (combination of lytic areas, sclerosis and new bone formation). 49 lesions (bone marrow edema, bone expansion, lytic areas and periosteal reaction) were verified by MRI in all eight children (4/8 children had WB MRI). Also, we found 17 asymptomatic lesions. Von Kalle et al. (21) concluded that WB MRI appeared to be more suitable as a major radiological criterion for the diagnosis of CRMO than radiographs, which was negative in $50 \%$ of cases. The WB MRI was the key in detecting subclinical lesions, which could assist in evaluating and excluding a pathological mass wherever in the body $(34,41)$.

How effective biopsy could be is still a debated topic, in fact histological features are not specific but it is very important to exclude any other causes of bone pain such as infectious osteomyelitis, a malignant bone tumor or $\mathrm{LCH}$. Some authors suggest that biopsy could be avoided if a child has classical radiological findings of CRMO, which fulfill the diagnostic criteria $(9,19,27)$. The authors of the Bristol diagnostic criteria (19) suggest that CRMO might be suspected in case of a bone pain with or without swelling and without significant features of infection, with the typical radiological findings (lytic areas, sclerosis, new bone formation) if the disease is multifocal; with no CRP level elevation if the disease affects one bone with CRP level greater than $30 \mathrm{~g} / \mathrm{L}$ and the bone biopsy shows inflammatory changes with no bacterial growth while not on antibiotic therapy (42). Accordingly, in our study children had characteristic radiologic lesions, and fulfilled other diagnostic criteria, so the diagnosis of CRMO was established; two biopsies were performed and showed inflammatory changes, but those children had CRP $<30 \mathrm{mg} / \mathrm{L}$. Gicchinal et al. (28) stated that twelve children in their study were without a biopsy, five of these had typical multiple lesions on MRI scan, one had a single affected area and had a sibling with typical CRMO, and three patients had a solitary lesion of the clavicle. Von Kalle et al. (21) diagnosis of CRMO was supported by histology in 37 of 53 patients, but in 16 patients without histologically proven osteomyelitis, the diagnosis was established according to the criteria proposed by Jansson et al. (9). In their study, Girschick et al. (11) found that frequent manifestations not directly related to bone involvement were mucocutaneous manifestations (5\% acne, 5\% palmoplantar pustulosis, $4 \%$ psoriasis, $3 \%$ papulopustular lesions, $2 \%$ urticarial rash) and gastrointestinal symptoms (8\%). Similar results were reported by Kaiser et al. (22). In their study skin lesions were present in $17 \%$ of children, three children presented with typical palmoplantar pustulosis, psoriasis or acne was observed in one child each, one child had both psoriasis and acne. The associated autoimmune disease was seen in 2/41 children: one boy had a diagnosis of unclassified panniculitis and one girl had an additional diagnosis of ANA-positive JIA with uveitis. No child had IBD. Our results were slightly different, $1 / 8$ children had pyoderma gangrenosum and acne conglobata, while one boy had granuloma recurrence on $\mathrm{n}$ his face. During the two-year follow-up, one boy developed IBD, and he also had an additional diagnosis of ANA positive JIA. Some authors consider CRMO the pediatric equivalent of SAPHO syndrome (synovitis, acne, pustulosis, hyperostosis, osteitis), characterized by association of osteoarticular and skin disorders with the age of onset ranging from adolescence to late adulthood (43). In our study, a boy who developed acnae conglobata was 9.8 years old, and his father with history of acnae vulgaris had no other characteristics, so they were not considered as having SAPHO syndrome. NSAIDs are the first choice which provide some degree of symptomatic relief and in up to $80 \%$ of patients prevent bone damage (43-45). Beck et al. (4) found that $43 \%$ of children treated with naproxen were symptom-free six months after starting treatment. The significant decrease in pain, functional impairment, swelling and over $50 \%$ of reduction in radiologically apparent lesions were noticed after 12 months. Our results were somewhat consistent with those; in $75 \%$ 
of children treatment with NSAIDs was sufficient to control the disease during the 8-month period. For three of them, who had progressions and new lesions on MRI at onset, treatment with naproxen was continued, and after 11 months the treatment was stopped since MRI showed regression of lesions and the children were symptoms free. On the other hand, in long-term follow-up study of 22 individuals with CRMO, only 9\% had a good response to NSAIDs, while $27.2 \%$ had no response at all (46). For treatment of children with CRMO refractory to NSAIDs monotherapy and/or with active spinal lesions, three consensus treatment plans (CTPs) were developed for the first 12 months of therapy. The three CTPs are methotrexate or sulfasalazine, TNF $\alpha$ inhibitors with optional MTX, and bisphosphonates. Short courses of glucocorticoids and continuation of NSAIDs are permitted for all regimens (44). So, Girschick et al. (11) reported partial response and remission together with bisphosphonates, NSAIDS, glucocorticoids and sulfasalazine, 91, 91, 92 and 87\%, respectively. Methotrexate and etanercept were considered less effective, particularly when compared with glucocorticoids and bisphosphonates, 71 and $71 \%$, respectively. The decision to escalate medical therapy must take into consideration the fact that most lesions are will resolve without significant sequelae, and spontaneous remission can occur $(10,30)$. Indications for escalation include persistent pain that affects normal activities, frequent recurrences, and functional limitations. Kerrison et al. (47) found significant pain relief and improved activity, and well-being with pamidronate use in seven children (three with spinal lesions) who failed to respond to NSAIDs. Simm et al. (48) and Miettunen et al. (49) also demonstrated the effectiveness of pamidronate in children with CRMO refractory to NSAIDs. Published data on the use of TNF $\alpha$ inhibitors in CRMO are more limited. A small cohort study by Eleftheriou et al. (50) showed decreased pain in children with $\mathrm{CNO}$ after infliximab treatment $(\mathrm{n}=3)$ and anakinra $(\mathrm{n}=1)$, later switched to adalimumab. Wipff et al. (51) observed the highest rates of clinical remission or efficacy (89\%) from TNF $\alpha$ inhibitors compared to glucocorticoids, methotrexate, sulfasalazine, and NSAIDs. Jansson et al. (52) reported disease remission induced by infliximab in two patients who failed to respond to NSAIDs, glucocorticoids, methotrexate and sulfasalzine and pamidronate. Borzutsky et al. (12) performed a retrospective analysis of 70 patients with CRMO and found that the estimated probability to response was $66 \%$ for sulfasalazine, $91 \%$ for methotrexate, 91\% for TNF $\alpha$ inhibitors and 95\% for corticosteroids. No individuals were treated with bisphosphonates. In our study, no children were treated with bisphosphonates either, but we had to escalate therapy in two children: 1 child was administered methotrexate and 1 child was administered sulfasalazine. Since the inflammatory process was not controlled with sulfasalazine, the TNF $\alpha$ inhibitor was introduced.

\section{The Limitations of the Study}

The major limitation of the study was the short time for observation. We presented the clinical outcomes of children in a short period of time for the assessment of the final outcome, recurrence of lesions. This can be evaluated many years after, not just after an initial follow up.

\section{Conclusions}

The diagnosis of CRMO is primarily based on exclusion of other differential diagnoses and, therefore, it is very important to have diagnostic tools such as the latest Bristol diagnostic criteria. Our results also confirm that the use of those diagnostic criteria may obviate the need for biopsy, shorten the time of diagnosis, save the bone from destruction, and avoid unnecessary treatments. However, it is important to improve the clinical understanding of CRMO by documenting more cases, route to diagnosis, and treatment choices made in order to help develop diagnostic and treatment guidelines.

Authors' Contributions: Conception and design: AĆ, DD and AS; Acquisition, analysis and interpretation of data: AĆ, AS, EZ, DD, MD and SS; Drafting the article: AĆ and SSD; 
Revising the article critically for intellectual content: AĆ, AS, DS, FDž, MD and SS; Approved final version of the manuscript: AĆ, SS, AS, EZ, SSD, DD, DS, MD and FDž.

Conflict of Interest: The authors declare that they have no conflict of interest.

\section{References}

1. Fergusson PJ, Sandu M. Current understanding of the pathogenesis and Management of Chronic Recurrent Multifocal Osteomyelitis. Curr Rheumatol Rep. 2012;14:13041.

2. Masters SL, Simon A, Aksentijevich I, Kastner DL. Horror autoinflammaticus:the molecular pathophysiology of autoinflammatory disease. Annu Rev Immunol. 2009;27:62-8.

3. McGonagle D, McDermott MF. A proposed classification of the immunological diseases. PLoS Med. 2006;3(8):297.

4. Stojanov S, Kastner DL. Familial autoinflammatory diseases: genetics, pathogenesis and treatment. Curr Opin Rheumatol. 2005;17(5):586-99.

5. Bjorksten B, Gustavson KH, Eriksson B, Lindholm A, Nordström S. Chronic recurrent multifocal osteomyelitis and pustulosis palmoplantaris. J. Pediatr. 1978;93(2):227-31.

6. Vittecoq O, Said LA, Michot C, Mejjad O, Thomine JM, Mitrofanoff P, et al. Evolution of chronic recurrent multifocal osteitis toward spondyloarthropathy over the long term. Arthritis Rheum. 2000;43(1):109-19.

7. Chun CS. Chronic recurrent multifocal osteomyelitis of the spine and mandible: case report and review of the literature, Pediatrics. 2004;113(4):380-4.

8. Hedrich CM, Hofmann SR, Pablik J. Morbach H, Girschick HJ. Autoinflammatory bone disorders with special focus on chronic recurrent multifocal osteomyelitis (CRMO). Pediatr Rheumatol. 2013;11:47.

9. Jansson A, Renner RD, Ramseur J, Mayer A, Haban M, Meindl A, et al. Classification of non-bacterial osteitis: retrospective study of clinical, immunological and genetic aspects in 89 patients. Rheumatology. 2007;46(1):15460.

10. Girschick HJ, Raab P, Surbaum S, Trust A, Kirschner S, Schneider P, et al. Chronic non-bacterial osteomyelitis in children. Ann Rheum Dis. 2005;64(2):279-85.

11. Girschick H, Finetti M, Orlando F, Schalm S, Insalaco A, Ganser G, et al. The multifaceted presentation of chronic recurrent multifocal osteomyelitis: a series of 486 cases from the Eurofever international registry. Rheumatology. 2018;57:1203-11.
12. Borzutzky A, Stern S, Reiff A, Zurakowski D, Steinberg EA, Dedeoglu F, et al. Pediatric chronic nonbacterial osteomyelitis. Pediatrics. 2012;130(5):1190-7.

13. Guerin-Pfyffer S, Guillaume-Czitrom S, Tammam S, Kone-Paut I. Evaluation of chronic recurrent multifocal osteitis in children by whole body magnetic resonance imaging. Joint Bone Spine. 2012;79(6):616-20.

14. Tlougan BE, Podjasek JO, O’Haver J, Cordova KB, Nguyen XH, Tee R, et al. Chronic recurrent multifocal osteomyelitis (CRMO) and synovitis, acne, pustulosis, hyperostosis, and osteitis (SAPHO) syndrome with associated neutrophilic dermatoses: a report of seven cases and review of the literature. Pediatr Dermatol. 2009;26:497505.

15. Schnabel A, Range U, Hahn G, Siepmann T, Berner R, Hedrich CM. Unexpectedly high incidences of chronic non-bacterial as compared to bacterial osteomyelitis in children. Rheumatol Int. 2016;36:1737-45.

16. Morbach H, Schneider P, Schwarz T, Hofmann C, Raab $\mathrm{P}$, Neubauer $\mathrm{H}$, et al. Comparison of magnetic resonance imaging and 99mTechnetium-labelled methylene diphosphonate bone scintigraphy in the initial assessment of chronic non-bacterial osteomyelitis of childhood and adolescents. Clin Exp Rheumatol. 2012;30:578-82.

17. Hofmann SR, Schnabel A, Rosen-Wolff A, Morbach H, Girschick HJ, Hedrich CM. Chronic nonbacterial osteomyelitis: pathophysiological concepts and current treatment strategies. J Rheumatol. 2016;43:1956-64.

18. Oligbu G, Jacobs B, Khan T. The Dilemma of Chronic Recurrent Multifocal Osteomyelitis. Reumatol Clin. 2019;18:30235-3.

19. Roderick MR, Shah R, Rogers V, Finn A, Ramanan AV. Chronic recurrent multifocal osteomyelitis (CRMO) advancing the diagnosis. Pediatr Rheumatol Online J. 2016;14:47.

20. Giedion A, Holthusen W, Masel LF, Vischer D. Subacute and chronic "symmetrical" osteomyelitis. Ann. Radiol. 1972;15(3):329-42.

21. von Kalle T, Heim N, Hospach T, Langendörfer M, Winkler P, Stuber T. Typical Patterns of Bone Involvement in Whole-Body MRI of Patients with Chronic Recurrent Multifocal Osteomyelitis (CRMO). Fortschr Röntgenstr. 2013;185:655-61.

22. Kaiser D, Bolt I, Hofer M, Relly C, Berthet G, Bolz $\mathrm{D}$, et al. Chronic nonbacterial osteomyelitis in children: a retrospective multicenter study. Pediatr Rheumatol Online J. 2015;13:25.

23. Costa-Reis P, Sullivan KE. Chronic recurrent multifocal osteomyelitis. J Clin Immunol. 2013;33(6):1043-56. 
24. Iyer RS, Thapa MM, Chew FS. Chronic recurrent multifocal osteomyelitis: review. Am J Roentgenol. 2011;196:87-91.

25. Kostik MM, Kopchak O, Chikova I, Isupova EMA. Comparison of different treatment approaches of pediatric chronic non-bacterial osteomyelitis. Rheumatol Int. 2019;39:89-96.

26. Acikgoz G, Averill LW. Chronic recurrent multifocal osteomyelitis: typical patterns of bone involvement in whole-body bone scintigraphy. Nucl Med Commun. 2014;35:797-807.

27. Falip C, Alison M, Boutry N, Job-Deslandre C, Cotten A, Azoulay R, et al. Chronicrecurrent multifocal osteomyelitis (CRMO): a longitudinal case series review. Pediatr Radiol. 2013;43(3):355-75.

28. Gicchino MF, Diplomatico M, Granato C, Capalbo D, Marzuillo P, Olivieri AN, et al. Giudice Chronic recurrent multifocal osteomyelitis: a case report. Ital J Pediatr. 2018;44:26.

29. Gikas PD, Islam L, Aston W, Tirabosco R, Saifuddin A, Briggs TW, et al. Nonbacterial osteitis: a clinical, histopathological, and imaging study with a proposal for protocol- based management of patients with this diagnosis. J Orthop Sci. 2009; 14(5):505-16.

30. Huber AM, Lam PY, Duffy CM, Yeung RS, Ditchfield M, Laxer D et al. Chronic recurrent multifocal osteomyelitis: clinical outcomes after more than five years of follow- up. J Pediatr. 2002;141(2):198-203.

31. Surendra AG, Shetty U. Chronic recurrent multifocal osteomyelitis: A rare entity. J Med Imaging Radiat Oncol. 2015;59(4):436-44.

32. Jurik AG, Moller S.H, Mosekilde L. Chronic sclerosing osteomyelitis of the iliac bone. Etiological possibilities. Skeletal Radiol.1988;17(2):114-8.

33. Jibri Z, Sah M, Mansour R. Chronic recurrent multifocal osteomyelitise mimicking osteoid osteoma. JBR-BTR. 2012;95(4):263-6.

34. Ferguson PJ, Sykelyk A, Figueredo R, Koropatnick J. Synergistic cytotoxicity against human tumor cell lines by oncolytic adenovirus dl1520 (ONYX-015) and melphalan. Tumori. 2016;102:31-9.

35. Catalano-Pons C, Comte A, Wipff J, Quartier P,Faye A, Gendrel D et al. Clinical outcome in children with chronic recurrent multifocal osteomyelitis. Rheumatology. 2008;47:1397-9.

36. Ferguson PJ, Lokuta MA, El-Shanti HI, Muhle L, Bing X, Huttenlocher A. Neutrophil dysfunction in a family with a SAPHO syndrome-like phenotype. Arthritis Rheum. 2008;58(10):3264-9.
37. Schnabel A, Range U, Hahn G, Siepmann T, Berner R, Hedrich CM. Unexpectedly high incidences of chronic non-bacterial as compared to bacterial osteomyelitis in children. Rheumatol Int. 2016;36:1737-45.

38. Probst FP, Bjorksten B, Gustavson KH. Radiological aspect of chronic recurrent Multifocal osteomyelitis. Ann Radiol. 1978;21:115-25.

39. Jurik AG. Chronic recurrent multifocal osteomyelitis. Semin Musculoskelet Radiol. 2004;8:243-53.

40. Hofmann SR, Kapplusch F, Girschick HJ, Morbach H, Pablik J, Ferguson PJ et al. Chronic Recurrent Multifocal Osteomyelitis (CRMO): Presentation, Pathogenesis, and Treatment. Curr Osteoporos Rep. 2017;15:542-54.

41. Duffy CM, Lam PY, Ditchfield M, Allen R, Graham HK. Chronic recurrent multifocal osteomyelitis: review of orthopaedic complications at maturity. J Pediatr Orthop. 2002;22:501-5.

42. Świdrowska-Jaros J, Smolewska E. A complicated path to the CRMO diagnosis-case of a 9year old girl whose story comes full circle. BMC Musculoskeletal Disord. 2019;20:392.

43. Schnabel AR, Range U, Hahn G, Berner R, Hedrich CM.Treatment response and longterm outcomes in children with chronic nonbacterial osteomyelitis. J Rheumatol. 2017;44:1058-65.

44. Zhao Y, Wu EY, Oliver MS, Cooper AM, Basiaga ML, Vora SS, et al. Consensus Treatment Plans for Chronic Nonbacterial Osteomyelitis Refractory to Nonsteroidal Antiinflammatory Drugs and/or With Active Spinal Lesions. Arthritis Care Res (Hoboken). 2018;70(8):1228-37.

45. Carr AJ, Cole WG, Roberton DM, Chow CW Chronic multifocal osteomyelitis, J. Bone Joint Surg. 1993;75(4):82-91.

46. Beck C, Morbach H, Beer M, Stenzel M, Tappe D, Gattelöhner et al. Chronic nonbacterial osteomyelitis in childhood: prospective follow-up during the first year of anti-inflammatory treatment, Arthritis Res Ther. 2010;12(2):74.

47. Kerrison C, Davidson JE, Cleary G, Beresford MW. Pamidronate in the treatment of childhood SAPHO syndrome. Rheumatology. 2004;43:1246-51.

48. Simm PJ, Allen RC, Zacharin MR. Bisphosphonate treatment in chronic recurrent multifocal osteomyelitis. J Pediatr. 2008;152:571-5.

49. Miettunen PM, Wei X, Kaura D, Reslan WA, Aguirre AN,Kellner JD. Dramatic pain relief and resolution of bone inflammation following pamidronate in 9 pediatric patients with persistent chronic recurrent multifocal osteomyelitis (CRMO). Pediatr Rheumatol Online J. 2009;7:2. 
50. Eleftheriou D, Gerschman T, Sebire N, Woo P, Pilkington CA, Brogan PA. Biologic therapy in refractory chronic nonbacterial osteomyelitis of childhood. Rheumatology. 2010;49:1505-12.

51. Wipff J, Costantino F, Lemelle I, Pajot C, Duquesne A, Lorrot $\mathrm{M}$, et al. A large national cohort of French patients with chronic recurrent multifocal osteitis. Arthritis Rheumatol. 2015;67:1128-37.

52. Jansson A, Renner ED, Ramser J, Mayer A, Haban M, Meindl A, et al. Classification of non-bacterial osteitis: retrospective study of clinical, immunological and genetic aspects in 89 patients. Rheumatology. 2007;46:154-60. 